\title{
The influence of spill-line geometry on a spray generated by a pressure- swirl atomizer
}

\author{
Milan Malý ${ }^{1, a}$, Lada Janáčková ${ }^{1}$, Jan Jedelský ${ }^{1}$ and Miroslav Jícha ${ }^{1}$ \\ ${ }^{1}$ Brno University of Technology, Faculty of Mechanical Engineering, Energy Institute, Technická 2896/2 Brno 616 69, Czech Republic
}

\begin{abstract}
An experimental investigation of characteristics of spray generated by a pressure-swirl atomizer (spill-return type) was performed using shadowgraphy and Phase-Doppler Anemometry (PDA). Several different geometries of the spill-return orifice were tested in terms of a spray stability and quality on a cold test bench. PDA measurement yields a drop-size distribution and velocity data while the shadowgraphy unveils a break-up process in detail. Performed measurements reveal significant differences in spray characteristics as well as differences in spray stability. The results suggest that the air core, formed inside the swirl chamber, passes through the spill orifice, which causes instability of the inner flow. These instabilities lead to a chaotic state of sheet breakup resulting in shortening of breakup distance. Obtained findings are used to propose possible changes in the atomizer design for improvement of its performance.
\end{abstract}

\section{Introduction}

A spill-return atomizer is basically a pressure-swirl atomizer that contains a bypass in the swirl chamber known as a spill-line (SL) orifice. The liquid is injected via tangential ports to the swirl chamber, where it is divided into two streams, one of them is discharged outside and atomized, the second one is "spilled" back to the reservoir throught the SL orifice. The amount of spilled fuel is regulated by a control valve in a spill-line. Fuel is always supplied to the swirl chamber at high pressure providing good atomization over a wide span of injection flow rate. This feature allows using the spillreturn atomizers in applications requiring the wide regulation range such as industrial burners and gas turbine combustors [1].

A number of studies focused on improvement of spray characteristics of pressure-swirl atomizers, but only a few of them were investigating spill-return atomizer and even less examined the influence of the spill-return orifice on the spray quality and stability. Nasr et al. in [2] tested several different diameters of spill orifice in terms of Sauter mean diameter (SMD) and flow rate. However, their atomizers were run with open spill line without any regulation, and the spill-feed ratio was controlled by a cross section of SL orifice. Our research is concentrated on test with regulated spill line along with closed spill line to simulate the full power of the atomizer.

Several small sized spill-return atomizers were chosen for this study varying only in the SL orifice geometry. Two different diameters of SL orifice were investigated with or without an obstacle blocking direct axial flow

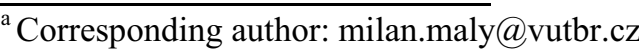

through the SL orifice. Also, several axial positions of the obstacle in the SL orifice were taken into account.

\section{Experimental setup}

Experiments were performed at a specially designed facility for cold spray generation under controlled conditions in the Spray laboratory at the Brno University of Technology.

\subsection{Cold test bench}

A schematic layout of the cold test bench is shown in Figure 1. It consists of a gear feed pump (3) that supplies fuel from a main tank (1) through filters (2), a flow meter (4), a pressure sensor (5), a temperature meter (7) and a control valve (8) into the atomizer (9). The spray falls into a collector and it is then returned to the main supply tank. The flow rate is controlled by a bypass needle valve (9). Spill-return line consist of a pressure sensor (6), a needle regulating valve (11) and a gear flow meter (12)

Flow rate in the fuel line is metered by Siemens Mass 2100 Di3 Coriolis mass flow meter fitted with a Mass 6000 transmitter. Flow rate uncertainty is $0.2 \%$ of its actual value. The uncertainty of pressure sensors (BD Senzor DMP 331i) is $0.35 \%$ of the actual value. The error of temperature sensor Omega PR-13 is $0.2{ }^{\circ} \mathrm{C}$.

Operation regimes of the atomizer were controlled by setting the inlet pressure to $0.5,1$ and $1.5 \mathrm{MPa}$ with the closed spill line by the valve near the atomizer. Spill mode of the atomizer was performed at an inlet pressure of $1 \mathrm{MPa}$ with the spill-feed ratio of 0.4 and 0.8 . The 
spill-fill ratio is defined as the ratio of the amount of

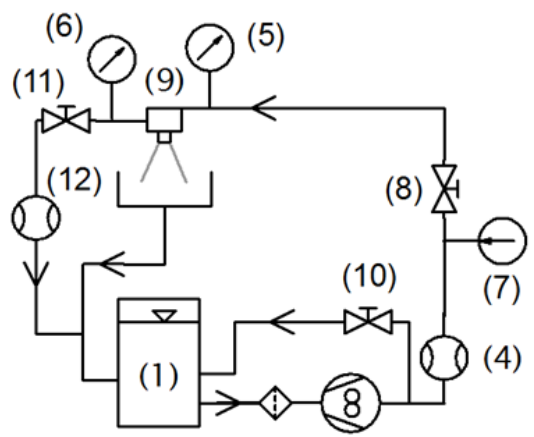

(2) (3)

Figure 1. Schematic layout of the experimental facility

spilled fuel to the amount of pumped fuel to the atomizer

Kerosene Jet A-1 was used as the testing liquid. Physical properties of Jet A-1 at room temperature are: surface tension $\sigma=0.029 \mathrm{~kg} / \mathrm{s}^{2}$, dynamic viscosity $\mu_{1}=0.0016 \mathrm{~kg} /(\mathrm{m} \cdot \mathrm{s})$ and density $\rho_{1}=795 \mathrm{~kg} / \mathrm{m}^{3}$. All tests were done with one batch at $25^{\circ} \mathrm{C}$.

\subsection{Phase Doppler anemometry}

Droplet sizes and velocities were probed by two component Phase Doppler anemometer (PDA) Dantec Dynamics A/S, see a schematic layout in Figure 2. PDA measurement was made at an axial distance $Z=25 \mathrm{~mm}$ from the exit orifice. One radial profile with 31 positions was measured. In each position, 35,000 samples or 15 second long measurement were taken. Detailed setup information is summarized into Table 1.

Spray images were taken by Canon EOS D70 with Canon EF $100 \mathrm{~mm} \mathrm{f} / 2.8$ UMS Macro lens fitted to $68 \mathrm{~mm}$ long extension tube. The spray was illuminated by Nd:YAG NewWave Research Gemini laser with a pulse duration of $5 \mathrm{~ns}$ and pulse energy of $5 \mathrm{~mJ}$. The imaging system was configured to capture a shadowgraphy images.

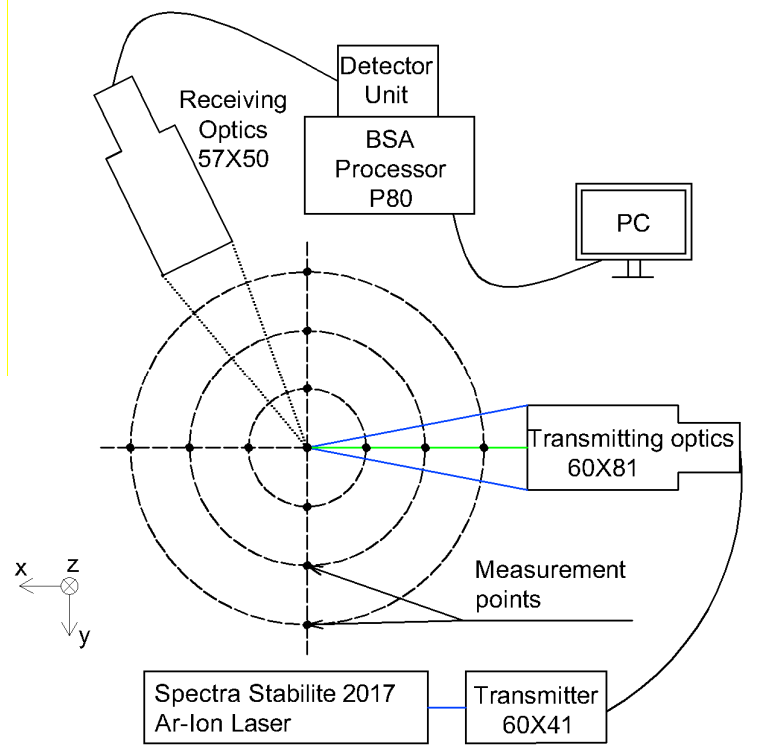

Figure 2. A setup of the PDA measurement
Table 1. PDA setup

\begin{tabular}{cc}
\hline Parameter & Value \\
\hline Laser power output & $1 \mathrm{~W}$ \\
Wavelength & $514.5 \mathrm{~nm}$ \\
$\begin{array}{c}\text { Front focal length of } \\
\text { transmitting optics }\end{array}$ & $310 \mathrm{~mm}$ \\
$\begin{array}{c}\text { Front focal length of } \\
\text { receiving optics }\end{array}$ & $800 \mathrm{~mm}$ \\
Scattering angle & $70^{\circ}$ \\
Mask & $\mathrm{B}$ \\
Spatial filter & $0.050 \mathrm{~mm}$ \\
Velocity & Axial \\
Velocity center & $8 \mathrm{~m} / \mathrm{s}$ \\
Velocity span & $32 \mathrm{~m} / \mathrm{s}$ \\
Sensitivity & $800 \mathrm{~V}$ \\
SNR & $0 \mathrm{~dB}$ \\
Signal gain & $20 \mathrm{~dB}$ \\
Level validation ratio & 8 \\
\hline
\end{tabular}

\subsection{Tested geometries}

Several small pressure-swirl atomizers with internal dimensions corresponding to

Figure 3 were investigated. Tested variations are listed in Table 2, where the $\mathrm{B}$ dimension represents a distance of the blocking obstacle from the top of the swirl chamber and $\mathrm{d}_{\mathrm{s}}$ a diameter of the SL orifice.

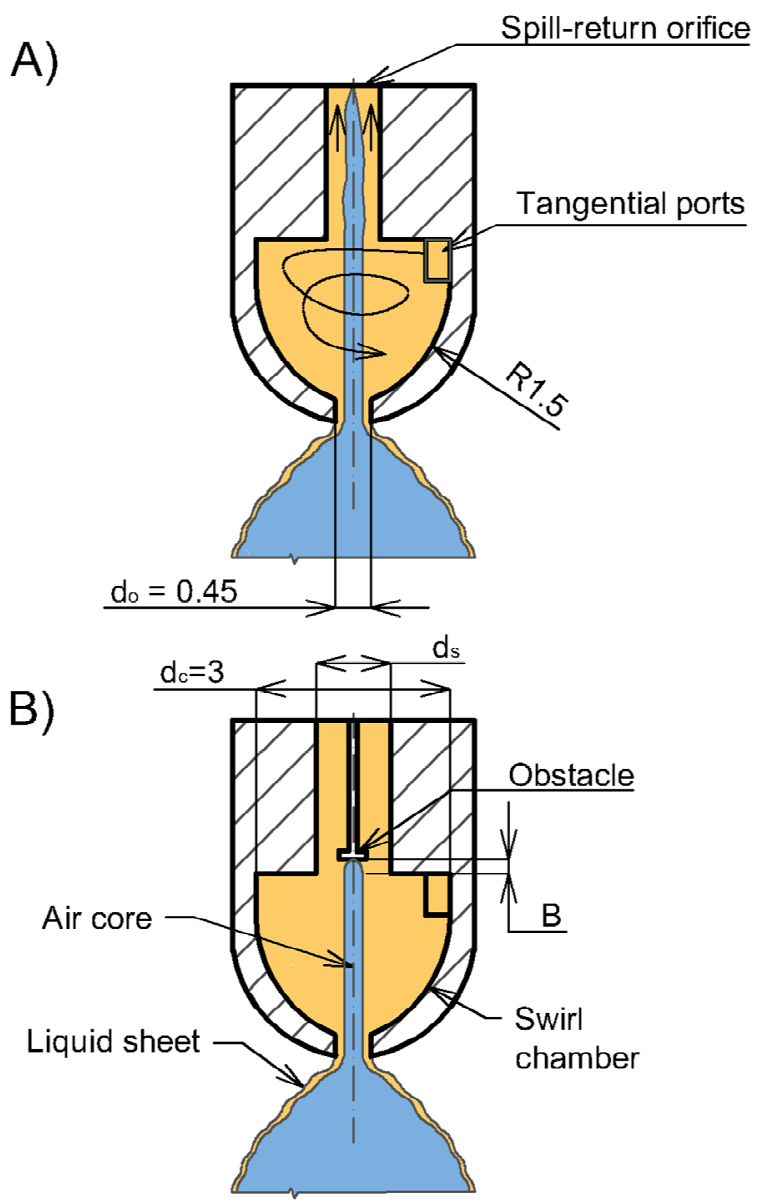

Figure 3. The geometry of the tested atomizer. A) Standard design (the SL orifice in the centre of the swirl chamber), B) Modified spill-line geometry with the obstacle 
Table 2. Tested atomizers configurations

\begin{tabular}{l|l|l} 
Configuration & B $[\mathbf{m m}]$ & $\mathbf{d}_{\mathbf{s}}[\mathbf{m m}]$ \\
\hline S1 & NA $^{*}$ & 0.8 \\
\hline S1B0 & 1 & 0.8 \\
\hline S1B2 & 3 & 0.8 \\
\hline S1B4 & 5 & 0.8 \\
\hline S2 & NA & 1.09 \\
\hline S2B0 & 1 & 1.09 \\
\hline S2B2 & 3 & 1.09 \\
\hline S2B4 & 5 & 1.09 \\
\hline *NA & Not &
\end{tabular}

*NA $=$ Not applied

\section{Results and discussion}

The first part of this chapter focuses to the flow characteristics such as mass flow rate, discharge coefficient and overpressure in the spill line, the second one deals with the liquid breakup and the third one is concerning PDA measurements.

\subsection{Discharge characteristics}

Measuring of liquid mass flow through the atomizers exit orifice and pressure on the spill line (with closed spill line) shows unexpected results. As it is clear from Figure 4 , liquid mass flow rate depends on the geometry of SL orifice even if the other dimensions remain unchanged. The lowest flow rate is almost the same for both SL orifice diameters $(0.8$ and $1.09 \mathrm{~mm})$ with the obstacle closest to the swirl chamber. Moderate flow rate is achieved by $0.8 \mathrm{~mm}$ SL orifice, where the flowrate is the same for all positions of the obstacle (expect the closest one) as well as for atomizer without the obstacle. Only at 1.5 MPa, the S1B4 show much higher flow rate than it would be expected and the reason of it is still unexplained. The 1.09 SL orifice (except the S2B0) have the highest flow rates. A difference about $40 \%$ in the mass flow rate between atomizers with and without obstacle shows a strong influence of SL orifice to the internal flow.

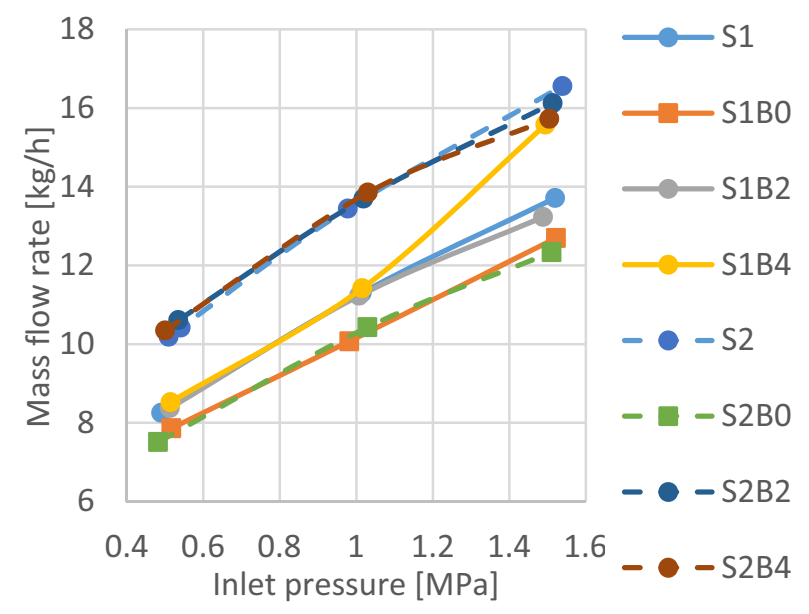

Figure 4. Mass flow rate with closed spill-line

Coefficient of discharge $\left(C_{\mathrm{D}}\right)$ is a ratio between the actual and the theoretical mass flow rate through the exit orifice, defined as:

$$
C_{D}=\frac{\dot{m}_{l}}{A_{0 \sqrt{2} \sqrt{2 p}}}
$$

The $C_{\mathrm{D}}$ is for pressure swirl atomizers usually low due to the presence of air core blocking off the central portion of the exit orifice and it is roughly independent on the inlet pressure. The $C_{\mathrm{D}}$ values as a function of inlet pressure are shown in Figure 5 for all tested atomizers. Figure 4 indicates main trends of $C_{\mathrm{D}}$ distribution over tested geometries. Atomizers with the obstacle closest to the swirl chamber have the lowest $C_{\mathrm{D}}$ varying from 0.47 to 0.44 . The highest $C_{\mathrm{D}}$ was reached with $1.09 \mathrm{~mm} \mathrm{SL}$ orifice diameter, changing from 0.64 to 0.57 .

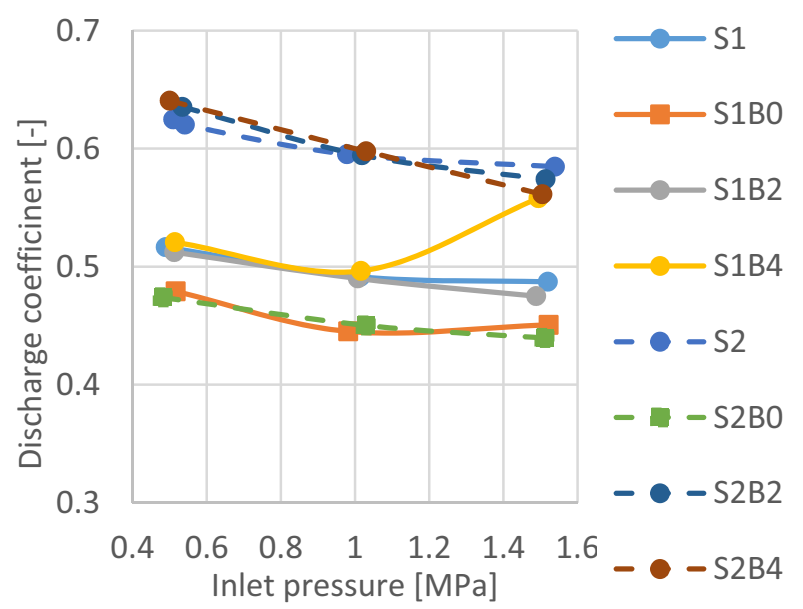

Figure 5. Discharge coefficient

A prediction of the discharge coefficient based on atomizer dimensions published by Rizk and Lefebvre in [3]:

$$
C_{D}=0.35\left(\frac{A_{p}}{d_{s} d_{o}}\right)^{0,5}\left(\frac{d_{s}}{d_{o}}\right)^{0,25}
$$

gives $C_{\mathrm{D}}=0.39$ and it is independent on inlet pressure and SL orifice dimensions.

Lee et al. [4] report $C_{\mathrm{D}}$ as a function of Reynolds number varying from 0.45 for high Re to 0.68 for low Re. They found a dependecy of air core stability on Re. Stable air core means a drop in the fuel flow rate as the center of the exit orifice is occupied by the air core.

Assuming that the air core created by internal swirl motion of tangentially injected liquid passes through the SL orifice, spill-return atomizer can be simplified into simple pressure-swirl type with extended swirl chamber. Influence of dimensions of the swirl chamber on internal flow was investigated by Kim et al. in [5]. They described a dependency of stability of the air core on the length of the swirl chamber, where longer swirl chamber lowers the air core stability. Taking these findings into account, we can assume that the geometries with the obstacle closest to the swirl chamber have stabilized the air core, while in the different configuration the air core is longer than its critical length and behave unstably, hence the $C_{\mathrm{D}}$ and the mass flow rate increases. 
The influence of spill-line pressure on the inlet pressure is revealed in Figure 6. As expected, SL pressure depends on the SL orifice diameter. The orifice with larger cross section has lower pressure losses and vice versa. But the obstacle in the spill line strongly increases the SL pressure (pressure drop is smaller). This behaviour is observed for both the SL orifice diameters it is attributed to the low $C_{\mathrm{D}}$ value.

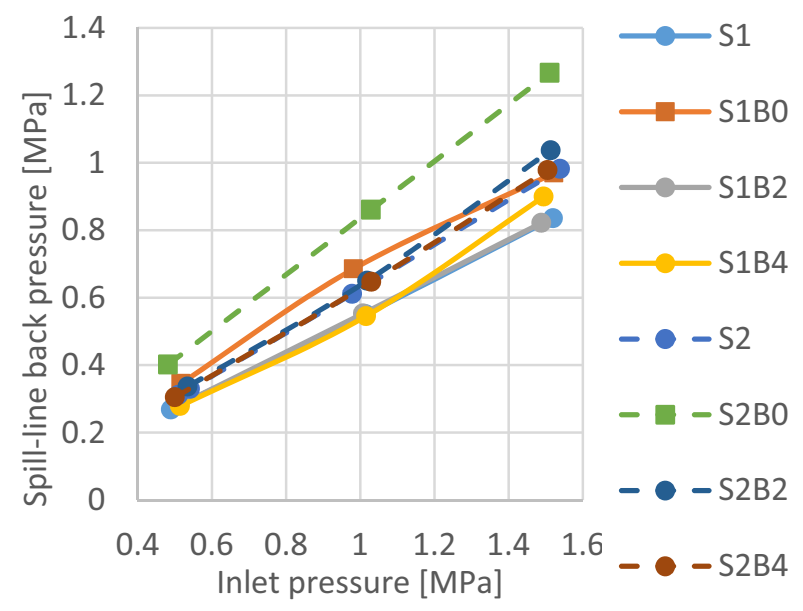

Figure 6. Spill-line back pressure

\subsection{Liquid breakup}

Droplet formation is a chaotic process, where discharged liquid sheet disintegrates due to aerodynamic forces between the liquid and stagnant surrounding air into filaments and ligaments and finally into drops in the form of a hollow cone spray. Longer breakup length would allow the liquid sheet to be thinned before the breakup and would result in smaller droplets. Entire breakup process is visualized in Figure 7. Images of the breakup process reveal how compact the liquid sheet is and if there are any long time instabilities.

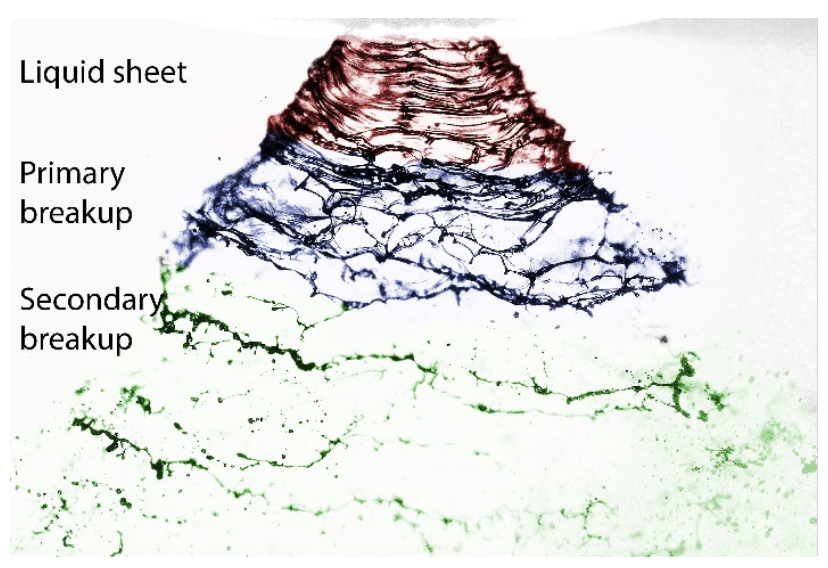

Figure 7. Near-nozzle spray structure

For detailed breakup, images see Figure 9. All tested configurations have in common that the breakup length strongly shortens with increasing inlet pressure, the atomizer S2B0 has the longest breakup length, which is most evident at $\Delta p=0.5 \mathrm{MPa}$ and $1.0 \mathrm{MPa}$, while the S1B0 does not appear to have similar nature and behave the same way as the configurations without the obstacle. This is in contrary to the findings from the previous chapter, where both the atomizer have exactly the same $C_{\mathrm{D}}$. Thus the same mode of the liquid break up was expected. Other atomizers (even those not shown in Figure 8) feature a chaotic breakup process where the liquid sheet is almost undetectable and very short. Short break up length might be linked to the instabilities of the air core that was predicted in the previous chapter.

\subsection{Spray characteristics}

Basic spray characteristics such as axial velocity profile, Sauter mean diameter profile or liquid mass distribution within the spray are evaluated at inlet overpressure 1 $\mathrm{MPa}$ with closed spill line. The configurations with the obstacle placed further from the exit orifice were omitted from the PDA measurements.

The radial distribution of the mean droplet axial velocity corresponds well to other observations of hollow cone sprays for the case of atomizers with the obstacle (see Figure 8), where axial velocity reaches a local maximum at approx. $16 \mathrm{~mm}$ from the spray centre. Geometries without the obstacle have this maximum much less distinct. Velocities in the spray centre are divided by the diameter of SL orifice, where $1.09 \mathrm{~mm}$ orifice (both, with and without the obstacle) have by $25 \%$ higher axial velocity than $0.8 \mathrm{~mm}$ orifice.

The SMD is lower for atomizers with the obstacle in the spray centre (see Figure 10). The difference between $\mathrm{S} 1$ and S1B0 is modest in all radial distances, however $\mathrm{S} 2$ have 2.5 times higher SMD in the centre compared to the same geometry with the obstacle (S2B0) which has the lowest SMD in almost every position.

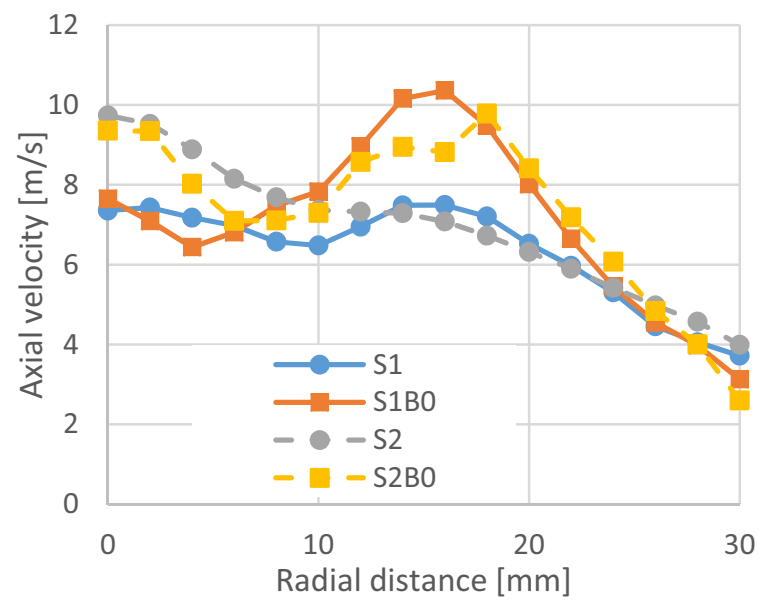

Figure 8. Axial velocity for $\Delta p=1 \mathrm{MPa}$ with closed spill line 

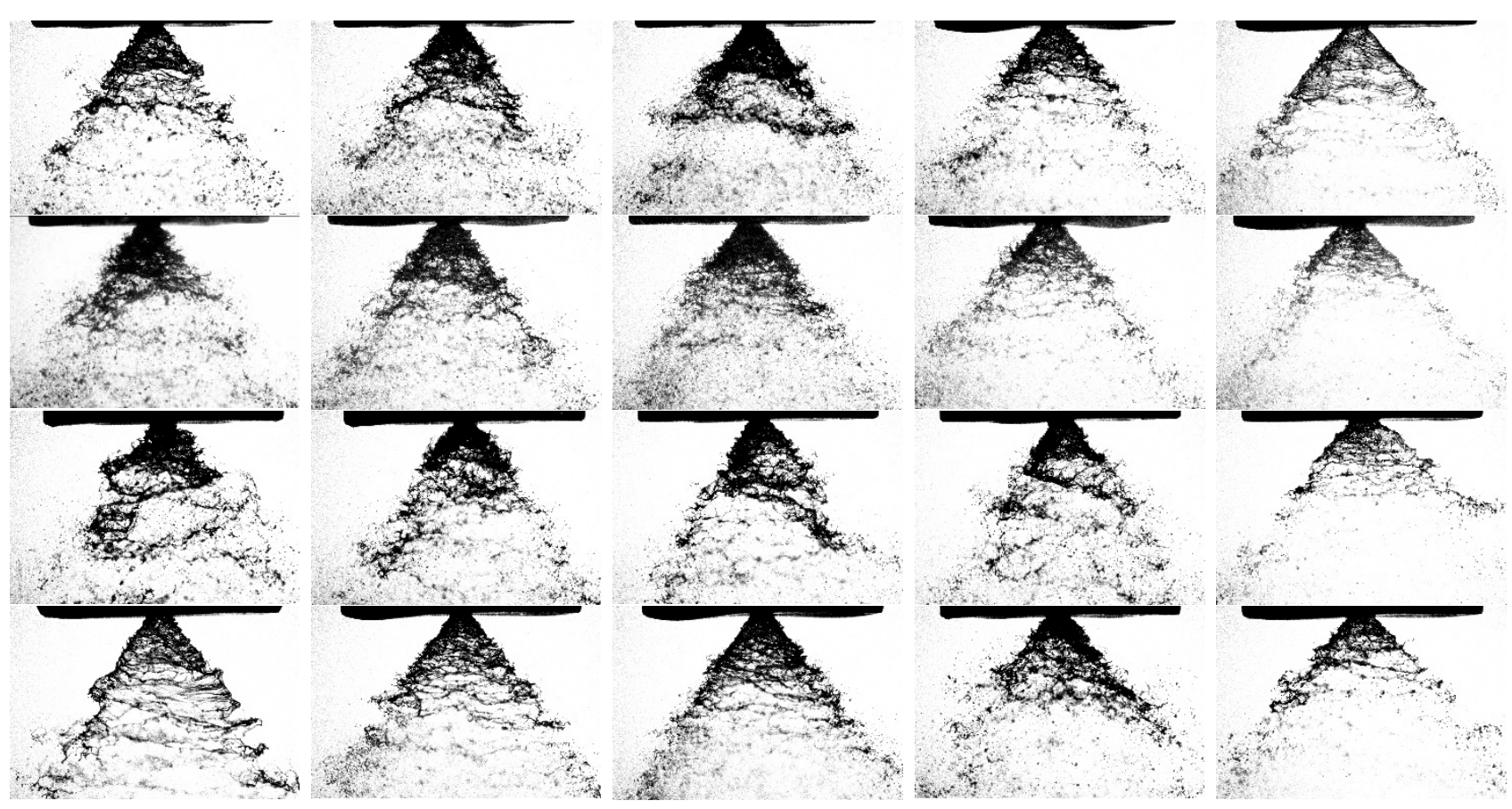

Figure 9. Liquid breakup visualization. Columns: from left $\Delta p=\mathbf{0 . 5}, 1,1.5 \mathrm{MPa}$ with SFR $0 ; 1 \mathrm{MPa}$ SFR 0.4 and $1 \mathrm{MPa}$ SFR 0.8 . Rows from top: S1, S1B0, S2, and S2B0. The size of each photograph is $7 \times 10 \mathrm{~mm}$.

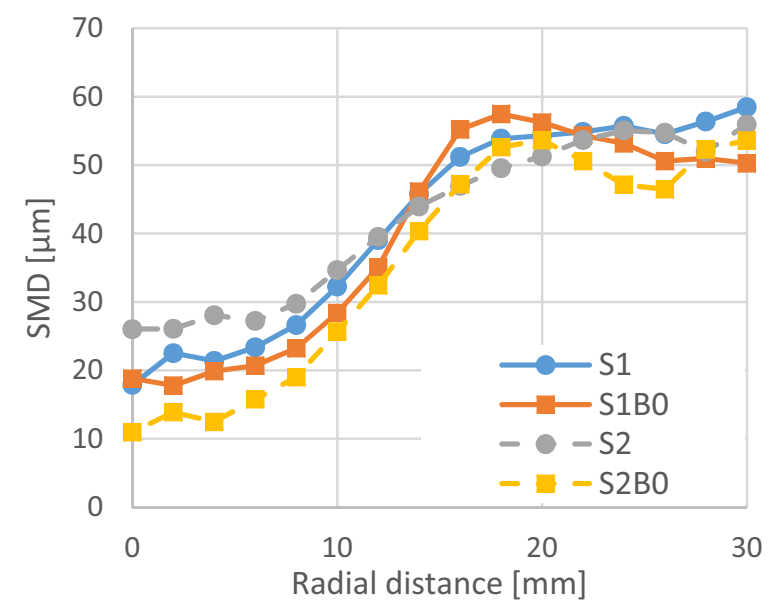

Figure 10. SMD for $\Delta p=1 \mathrm{MPa}$ with closed spill line

Cumulative liquid mass distribution in Figure 11 reveals significant divergence between the configurations with and without the obstacle. S1B0 and S2B0 have more liquid concentrated at positions further from spray centreline - where an annular liquid sheet is expected. Atomizers without the obstacle have concentrated about $37 \%$ of entire liquid mass flow at radial distance of $11 \mathrm{~mm}$ while the atomizers with the obstacle only $16 \%$ thus the mass distribution of S1 and S2 does not fully correspond to the idea of hollow cone spray.

This observation together with findings from Figure 8 indicate a problem of the liquid sheet stability that was confirmed by the breakup visualization in Figure 9.

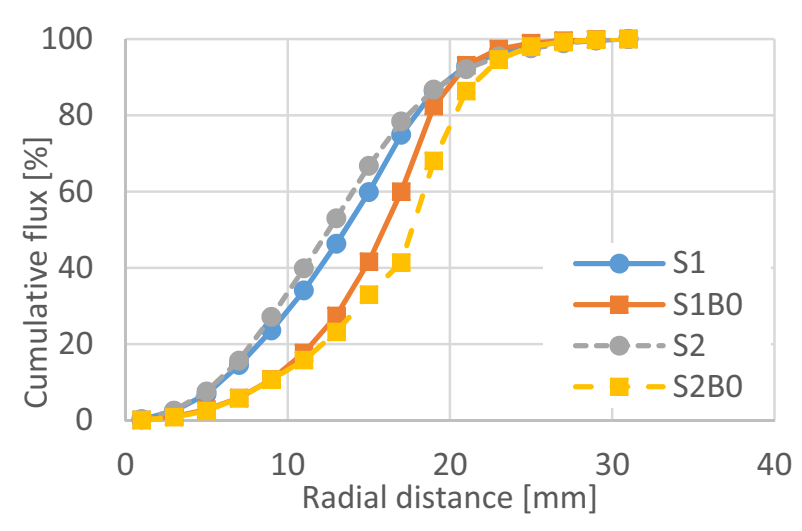

Figure 11. Liquid mass distribution $\Delta p=1 \mathrm{MPa}$

For evaluation of inlet pressure influence on SMD a global form of the SMD was calculated:

$$
I S M D=\frac{\sum_{i=1}^{N_{i}} n_{i} f_{i} D_{i}^{3}}{\sum_{i=1}^{N_{i}} n_{i} f_{i} D_{i}^{2}}
$$

As shown in Figure 12, with increasing pressure the ISMD decreases for all tested atomizers. The highest ISMD in the whole tested range is attributed to the S1B0 while the S2B0 has about 10\% lower ISMD. Both atomizers have the same $C_{\mathrm{D}}$, but the disparity in their ISMD confirms a different breakup mode showed in Figure 9. The lowest ISMD at $0.5 \mathrm{MPa}$ was reached by the S2B0 as it was predicted by the visualization (the longest breakup) however the S2 has the lowest ISMD at the rest of the tested regimes. 


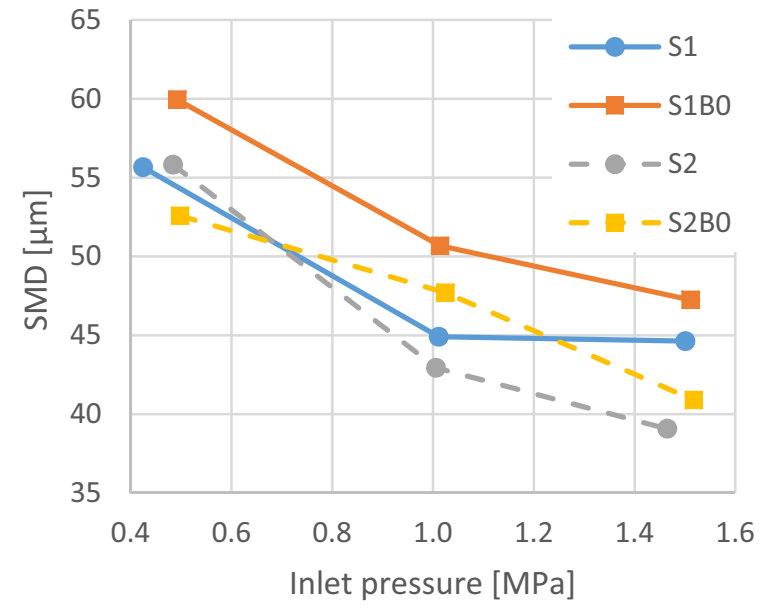

Figure 12. Global SMD as function of inlet pressure

\section{Conclusion}

The study of the influence of various SL orifice on spray was focused on discharge characteristics, near nozzle liquid break up and spray quality in terms of liquid distribution and SMD. Discharge coefficient of the flow through the exit orifice was found dependent on the diameter of SL orifice and also on the obstacle presence. A question of the air core instabilities caused by air core passing through the SL orifice were discussed with other related publications.

Spray visualization shows very short and chaotic breakup except the atomizer with the obstacle close to the swirl chamber and large diameter of SL orifice.

The spray structure was described based on spatially resolved velocity, drop size and mass flux. Different radial distributions of axial velocity indicate a problem with the hollow cone liquid sheet formation in the case of atomizers without the obstacle which is also confirmed by the analysis of liquid mass distribution across the spray diameter.

This study is a brief introduction to the problem of the influence of SL orifice geometry on the spray characteristics and reveals the importance of proper SL orifice design. Further investigation with a focus on the internal flow shows its necessity due to a lack of published papers dealing with phenomena.

\section{Acknowledgement}

This work has been supported by the project No. GA1509040S funded by the Czech Science Foundation, the project LO1202 NETME CENTRE PLUS with the financial support from the Ministry of Education, Youth and Sports of the Czech Republic under the "National Sustainability Programme I" and project Reg. No. FSI-S14-2355 funded by the Brno University of Technology.
3. N. K. Rizk, and A. H. Lefebvre, Journal of Propulsion and Power, 1, 193-199 (1985)

4. E. J. Lee, S. Y. Oh, H. Y. Kim., Experimental thermal and fluid science, 34, 1475-1483 (2010)

5. S. Kim, T. Khil, D. Kim, Measurement Science and Technology, 20 (2009)

\section{References}

1. A. H. Lefebvre, Atomization and sprays (1989)

2. G. Nasr, A. Yule, and S. Lloyd, ILASS (2007) 